\title{
某些无机盐在 $\mathrm{ZnO}-\mathrm{H}_{2} \mathrm{O}$ 分散体系中 光反应的研究 ${ }^{*}$
}

\author{
陈次平任新民 \\ (中国科学院感光化学研究所, 北京100101) \\ 陆道惠。张永康 \\ (中国科学院化学研究所, 北京 100080)
}

关镜词自旋捕捉、自由基、氧化锌

众所周知, 含半导体粉末的多相体系吸收了一定能量的光子, 便会在半导体的导带与价带 中产生电子空穴对. 它们与半导体周围的介质以各种方式发生电何转移而产生各种不同的活 泼中间体. 这类光化学反应与太阳能利用、光催化、污物的光降解以及成像体系有关, 因此引 起人们极大的关注.

Aurian-Blajeni 等 ${ }^{[1]}$ 曾用自旋捕捉技术研究了含碳酸氢钠的 $\mathrm{WO}_{3}-\mathrm{H}_{2} \mathrm{O}$ 悬浮液中光反应 产生的自由基中间体，观察到 $\cdot \mathrm{OH}, \cdot \mathrm{CO}_{3}^{-}, \cdot \mathrm{H}, \cdot \mathrm{CH}_{2} \mathrm{OH}, \cdot \mathrm{CO}_{2}^{-}$自由基加合物的 ESR 信 号.

因为自旋捕捉剂 5,5-二甲基-1-吡咯啉- $\mathrm{N}$-氧化物 (5,5-Dimethyl-1-pyrroline-N-oxide, DMPO) 的加合物的超精细分裂常数显著地依赖于被捕自由基的性质, 变化幅度较大, 而且它 的水溶性也好. 我们用它研究了含亚硫酸钠、亚硫酸氢钠、偏重亚硫酸钠、过硫酸盐的 $\mathrm{ZnO}$ $\mathrm{H}_{2} \mathrm{O}$ 分散体系中光反应产生的自由基中间体.

\section{一、实 验}

1. 试剂 5,5-二甲基-1-吡咯啉- $\mathrm{N}$-氧化物（DMPO）按文献[2]合成. 氧化锌及亚硫 酸钠、亚硫酸氢钠、偏重亚硫酸钠、过硫酸钠、过硫酸钾、过硫酸铵均为分析纯试剂。水为二次 蒸馏水.

2.ESR 波谱所用仪器为德国 Bruker 公司的 ESP 300 型 ESR 波谱仪. 室温下用 扁平池测谱. 在 $50 \mathrm{mg} \mathrm{ZnO}$ 粉末中加人 $5 \mathrm{ml} \mathrm{H}_{2} \mathrm{O}$ 用超声发生器进行分散, 然后取 $0.25 \mathrm{ml}$ 分散 体加人 DMPO $(0.05 \mathrm{~mol} / \mathrm{L})$ 和各种无机盐 $(0.5-1.0 \mathrm{~mol} / \mathrm{L})$ 用 GCQ-200 型高压录灯 照 射至 ESR 信号最强(用 $350 \mathrm{~nm}$ 的滤光片除去 uv 线). 测谱前样品通高纯氮除氧.

\section{二、结果与讨论}

1. $\cdot \mathrm{OH}$ 自由基 含 $\mathrm{DMPO}$ 的 $\mathrm{ZnO}-\mathrm{H}_{2} \mathrm{O}$ 悬浮液光照时立即观察到强度 为 $1: 2: 2: 1$

本文 1990 年 4 月 14 日收到. 1990 年 5 月 31 日收到修改稿.

* 北京分子动态及稳态结构化学国家实验室资助课题. 
的四重峰的ESR信号, 其超精细分裂常数 $a_{\mathrm{N}}=a_{\mathrm{H}}=14.9 \times 10^{-4} \mathrm{~T}$, 这是 DMPO 与 $\cdot \mathrm{OH}$ 自 由基的加合物的特征谱 ${ }^{[3]}$.

已知, 当 $\mathrm{ZnO}$ 吸收了波长 $\lambda \leqslant 380 \mathrm{~nm}$ 的光子时, 电子便从其价带跃迁到导带, 而在其 价带留下空穴. 这些电子和空穴将在 $\mathrm{ZnO}$ 和溶剂的界面上发生氧化还原反应. 如溶剂为 $\mathrm{H}_{2} \mathrm{O}$ 时, $\mathrm{H}_{2} \mathrm{O}$ 被空穴氧化而产生了 $\cdot \mathrm{OH}_{2}$ 自由基.

$$
\begin{aligned}
& \mathrm{ZnO}+\mathrm{h} v \rightarrow \mathrm{h}^{+}+\mathrm{e}^{-} \\
& \mathrm{H}_{2} \mathrm{O}+\mathrm{h}^{+} \rightarrow \cdot \mathrm{OH}+\mathrm{H}^{+}
\end{aligned}
$$

2. $\cdot \mathrm{SO}_{3}^{-}$自由基 含 $\mathrm{DMPO}$ 和 $\mathrm{Na}_{2} \mathrm{SO}_{3}$ 的 $\mathrm{ZnO}-\mathrm{H}_{2} \mathrm{O}$ 悬浮液光照时立即出现如图 1 所示的 $3 \times 2$ 重峰的 ESR 谱, 其超精细分裂常数 $a_{\mathrm{N}}=14.49 \times 10^{-4} \mathrm{~T}, a_{\mathrm{H}}=16.28 \times 10^{-4} \mathrm{~T}$. 这是 DMPO 与 $\mathrm{SO}_{3}^{-}$自由基加合物的 ESR 谱 ${ }^{[4]}$.

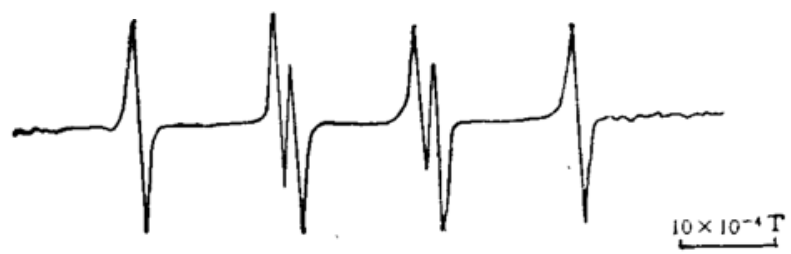

图 1 含 DMPO 和 $\mathrm{Na}_{2} \mathrm{SO}_{3}$ 的 $\mathrm{ZnO}_{\mathrm{n}} \mathrm{H}_{2} \mathrm{O}$ 分散体系光照得到的 ESR 谱

如果用 $\mathrm{NaHSO}_{3}$ 或 $\mathrm{Na}_{2} \mathrm{~S}_{2} \mathrm{O}_{5}$ 代替 $\mathrm{Na}_{2} \mathrm{SO}_{3}$ 重复同样的实验, 也得到了类似图 1 的 ESR 谱. 它们的超精细分裂常数列在表 1 中.

- $\mathrm{SO}_{3}^{-}$的产生是由这些盐与 $\mathrm{ZnO}-\mathrm{H}_{2} \mathrm{O}$ 悬浮液光照时产生的 $\cdot \mathrm{OH}$ 自由基或光生空穴作用 所致。

$$
\begin{aligned}
& \mathrm{SO}_{3}^{=}+\cdot \mathrm{OH} \longrightarrow \cdot \mathrm{SO}_{3}^{-}+\mathrm{OH}^{-}, \\
& \mathrm{SO}_{3}^{=}+\mathrm{h}^{+} \longrightarrow \cdot \mathrm{SO}_{3}^{-}, \\
& \mathrm{HSO}_{3}^{-}+\cdot \mathrm{OH} \longrightarrow \cdot \mathrm{SO}_{3}^{-}+\mathrm{H}_{2} \mathrm{O}, \\
& \mathrm{HSO}_{3}^{-}+\mathrm{h}^{+} \longrightarrow \cdot \mathrm{SO}_{3}^{-}+\mathrm{H}^{+}, \\
& \mathrm{S}_{2} \mathrm{O}_{5}^{=}+2 \cdot \mathrm{OH} \longrightarrow 2 \cdot \mathrm{SO}_{3}^{-}+\mathrm{H}_{2} \mathrm{O} .
\end{aligned}
$$

3. $\mathrm{SO}_{4}^{-}$自由基 含 $\mathrm{Na}_{2} \mathrm{~S}_{2} \mathrm{O}_{8}$ (或 $\mathrm{K}_{2} \mathrm{~S}_{2} \mathrm{O}_{8},\left(\mathrm{NH}_{4}\right)_{2} \mathrm{~S}_{2} \mathrm{O}_{3}$ ) 与 DMPO 的水溶液进行光照 $(\lambda \geqslant 350 \mathrm{~nm})$ 时, 观察到如图 $2 \mathrm{a}$ 所示的 $\mathrm{ESR}$ 谱. 它清楚地表明有・OH 自由基加合物产生, 同时有分辨不好的 $\mathrm{SO}_{4}^{-}$加合物存在. 这图谱相当稳定, 在光照 $15 \mathrm{~min}$ 内信号强度及两种 加合物的比值的变化都很小. 这个结果与 Harbour 和 Hair 在含 DMPO 的 $\mathrm{Na}_{2} \mathrm{~S}_{2} \mathrm{O}_{8}$ 溶液 进行紫外光照时观察到的结果 ${ }^{[3]}$ 有些相似. 他们观察到 $\mathrm{SO}_{4}^{-}$自由基加合物的信号在 $\mathrm{Na}_{2} \mathrm{~S}_{2} \mathrm{O}_{3}$ 光解的几秒钟内产生并很快衰减. 同时观察到有少量的. $\mathrm{OH}$ 自由基加合物存在. 他们认为 这两种自由基可能是由以下反应产生的 ${ }^{[6]}$ :

$$
\begin{gathered}
\mathrm{S}_{2} \mathrm{O}_{8}^{*} \stackrel{h \nu}{\longrightarrow} 2 \mathrm{SO}_{4}^{-}, \\
\mathrm{SO}_{4}^{-}+\mathrm{OH}^{-} \longrightarrow \mathrm{SO}_{4}^{-}+\cdot \mathrm{OH}^{-} .
\end{gathered}
$$

如何解释在我们的实验条件下所得的结果呢? 我们认为 $\mathrm{Na}_{2} \mathrm{~S}_{2} \mathrm{O}_{3}$ 溶液光照产生的 $\mathrm{SO}_{4}^{-}$ 自由基很可能与 $\mathrm{H}_{2} \mathrm{O}$ 作用发生以下的反应 ${ }^{[7]}$ :

$$
\mathrm{SO}_{4}^{-\cdot}+\mathrm{H}_{2} \mathrm{O} \rightleftharpoons \cdot \mathrm{OH}+\mathrm{HSO}_{4}^{-} \text {, }
$$



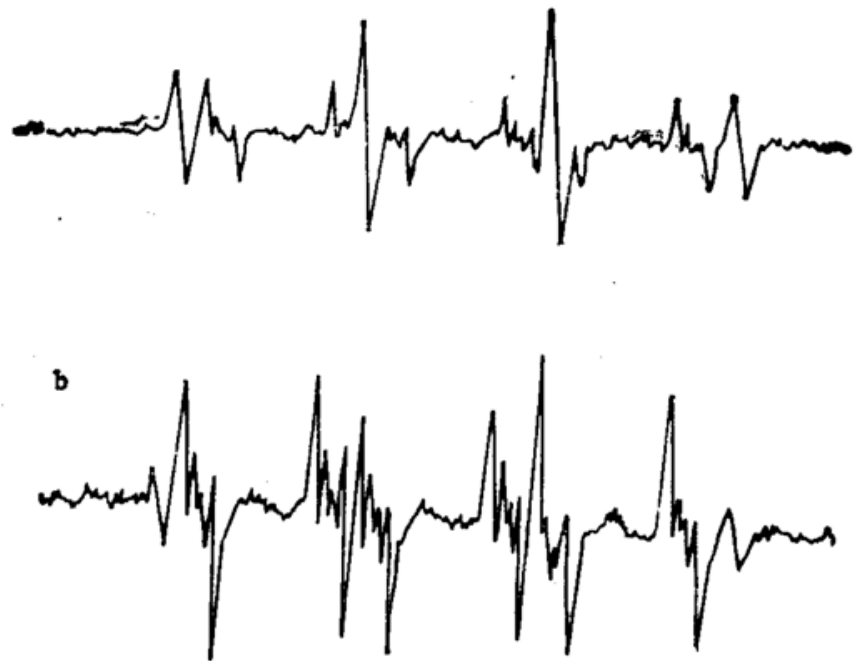

图 2 含 DMPO 的 $\mathrm{Na}_{2} \mathrm{~S}_{2} \mathrm{O}_{8}$ 水溶液光照得到的 ESR 谱 a. 无 $\mathrm{ZnO}$; b. 有 $\mathrm{ZnO}$

因此 $\mathrm{SO}_{4}^{-}$自由基与 $\cdot \mathrm{OH}$ 自由基加合物信号的相对强度能保持相当稳定.

为了进一步印证以上的设想, 我们进行了如下的实验：当含 DMPO 的 $\mathrm{ZnO}-\mathrm{H}_{2} \mathrm{O}-\mathrm{Na}_{2} \mathrm{~S}_{2} \mathrm{O}_{8}$ 体系在同样条件下进行光照时, 观察到如图 $2 \mathrm{~b}$ 所示的 ESR 谱. 由图可见, $\cdot \mathrm{OH}$ 自由基加合 物的信号逐渐减弱, 而 $\mathrm{SO}_{4}^{--}$自由基加合物的信号逐渐增强,而且分辨得很好.

本文上面已提及 $\mathrm{ZnO}$ 水溶液进行光照时会有 $\cdot \mathrm{OH}$ 自由基产生. 因 此在含 $\mathrm{ZnO}$ 的 $\mathrm{Na}_{2} \mathrm{~S}_{2} \mathrm{O}_{8}$ 溶液进行光照时, 使原来均相中的平衡反应(10)右部的・ $\mathrm{OH}$ 自由基浓度增加, 致使平 衡向左偏移. 因而 $\mathrm{SO}_{4}^{-}$自由基的浓度增加, 其自由基加合物的浓度也相应增加. 同时垱体 系进行光照时, 在 $\mathrm{ZnO}$ 的导带上发生了以下的反应 ${ }^{[3]}$ :

$$
\begin{aligned}
& \mathrm{O}_{2}+\mathrm{e}^{-} \longrightarrow\left(\mathrm{O}_{2}^{-}\right)_{s}, \\
& \left(\mathrm{O}_{2}^{-}\right)_{s}+\mathrm{H}^{+} \rightleftharpoons\left(\mathrm{HO}_{2}\right)_{s}, \\
& \left(\mathrm{HO}_{2}\right)_{s}+\mathrm{e}^{-} \longrightarrow\left(\mathrm{HO}_{2}^{-}\right)_{s}, \\
& \left(\mathrm{HO}_{2}^{-}\right)_{s}+\mathrm{H}^{+} \rightleftharpoons\left(\mathrm{H}_{2} \mathrm{O}_{2}\right)_{3} \rightleftharpoons \mathrm{H}_{2} \mathrm{O}_{2} .
\end{aligned}
$$

\begin{tabular}{|c|c|c|c|c|c|}
\hline 添加剂 & $a_{N}\left(\times 10^{-4} \mathrm{~T}\right)$ & $a_{H}^{8}\left(\times 10^{-4} \mathrm{~T}\right)$ & $a_{H}^{r_{1}}\left(\times 10^{-4} \mathrm{~T}\right)$ & $a_{\mathrm{H}}^{\gamma_{2}}\left(\times 10^{-4} \mathrm{~T}\right)$ & 自由基 \\
\hline $\begin{array}{l}\mathrm{H}_{2} \mathrm{O} \\
\mathrm{Na}_{2} \mathrm{SO}, \\
\mathrm{Na}_{2} \mathrm{HSO}, \\
\mathrm{Na}_{2} \mathrm{~S}_{2} \mathrm{O}_{3} \\
\mathrm{Na}_{2} \mathrm{~S}_{2} \mathrm{O}_{8}\end{array}$ & $\begin{array}{l}14.90 \\
14.49 \\
14.47 \\
14.49 \\
13.78 \\
14.93\end{array}$ & $\begin{array}{l}14.90 \\
16.28 \\
16.18 \\
16.28 \\
10.16 \\
14.93\end{array}$ & 1.40 & 0.89 & $\begin{array}{l}\cdot \mathrm{OH}^{-} \\
. \mathrm{SO}_{3}^{-} \\
. \mathrm{SO}_{3}^{-} \\
. \mathrm{SO}_{i}^{-} \\
\mathrm{SO}_{i} \cdot \\
. \mathrm{OH}\end{array}$ \\
\hline
\end{tabular}

表 1 含某些无机盐的 $\mathrm{ZnO}_{\mathrm{n}} \mathrm{H}_{2} \mathrm{O}-\mathrm{DMPO}$ 体系光照时所得结果

众所周知, 体系中氧的存在会使 ESR 谱的谱线加宽, 使信号难以分辨. 由于 $\mathrm{ZnO}$ 上导 带电子转移到被吸附的 $\mathrm{O}_{2}$ 中产生了 $\mathrm{O}_{2}^{-}$(方程11), 这样体系中 $\mathrm{O}_{2}$ 被消耗, 使 $\mathrm{SO}_{4}^{-}$自由 基加合物的信号分辨得很好.

有趣的是, 当体系进行光照 $15 \mathrm{~min}$ 时, 观察到的 ESR 谱中 - OH 自由基加合物的信号完 
全消失, $\mathrm{SO}_{4}^{-}$自由基加合物的信号相对减弱并有新的难以鉴别的自由基加合物的信号出现.

$$
\cdot \mathrm{OH}+\mathrm{H}_{2} \mathrm{O}_{2} \longrightarrow \mathrm{H}_{2} \mathrm{O}+\mathrm{HO}_{2},
$$

这表明・ $\mathrm{OH}$ 自由基还可能有其它(如方程 15 所示)的反应发生 ${ }^{[7]}$, 尚待进一步研究.

\section{参考文献}

[1] Aurian-Blajeni, B., Halmann. M., Manassen, J., Photochem. Photobiol., 35(1982), 157.

[2] Bonnett, R., Brown, R. F. C., Clark. V. M., Sutherland, I. D., Todd, A. R., J. Chem. Soc. 1959. 2094 .

[3] Harbour, J. R., Hair, M. L., J. Phys. Chem., 83(1979), 552.

[4] Chignell, C. F., Kalyanaraman, B., Sik, R. H., Mason. R. P.. Photochem. Photobiol., 34(1981), 147.

[5] Harbour, J. R., Hair, M. L.. Can. J. Chem., 57(1979), 1150,

[6] Chawla, O. P., Fessenden, R. W., J. Phys. Chem., 79(1975), 2593.

[7] Tang, Y., Thorn, R. P., Mauldin 111, R. L., Wine, P. H., J. Photochem. Photobiol. A., 44 (1988). 243. 